\title{
石墨悕材料在水处理中的应用: 传质机制与吸附特性
}

\author{
邓会 ${ }^{(12)}$, 孙鹏展 ${ }^{(2)}$, 张迎九 ${ }^{(1)}$, 朱宏伟 ${ }^{2 *}$ \\ (1) 郑州大学物理系, 郑州 450052; \\ (2) 清华大学材料学院, 新型陶瓷与精细工艺国家重点实验室, 北京 100084 \\ * 联系人, E-mail: hongweizhu@tsinghua.edu.cn
}

2015-06-26 收稿, 2015-08-12 接受, 2015-09-17 网络版发表

\begin{abstract}
摘要 石墨烯由于独特的单原子层二维结构和高比表面积等优异性能而被用作选择性分离膜和 吸附剂, 在水处理领域具有潜在的应用前景. 本文综述了石墨烯纳米多孔膜和层状堆叠的氧化 石墨烯渗透膜对气体、水及离子的传质行为. 纳米多孔膜因其制备技术和不成熟的打孔技术等 原因而具有一定局限性; 而层状渗透膜由于制备方法简单、成本低、高通透性和高选择性等优 点, 在水净化领域具有广阔的应用空间。进一步综述了石墨烯吸附材料对水中重金属离子、染料 和有机污染物的吸附行为, 分析了石墨烯材料表面官能团与污染物的相互作用机理. 最后展望 了石墨烯材料在膜分离、海水淡化和污染物去除等环境应用中的机遇和挑战.
\end{abstract}

关键词

石墨烯

氧化石墨烯

传质

吸附

水处理
水资源短缺是当今全球面临的最大挑战之一, 地球 $70 \%$ 被海水覆盖和严重的水污染, 因此海水淡 化和污水处理日益引起人们的关注. 纳米材料的快 速发展对于传统膜材料具有重要的推动作用. 例如, 纳米材料提供的亚纳米级通道为水分子提供了高速 传输通道, 且阻止大分子的通过 ${ }^{[1]}$, 从而能够实现海 水的脱盐与淡化.

石墨烯 (graphene)作为纳米材料之一, 自被发现 以来, 由于其独特的单原子层二维结构和优异的性 能, 如高比表面积、优异的电学、热学、光学和力学 等而受到广泛关注 ${ }^{[2,3]}$. 尤其在关于纳米通道或纳米 孔传质现象的研究中 ${ }^{[4,5]}$, 石墨烯由于 $\mathrm{sp}^{2}$ 杂化的碳原 子紧密排列成蜂巢状晶体结构而显示出特殊的传质 特性. 石墨烯薄膜基体上可引人纳米孔或人工设计 堆积结构, 进而作为一种高通透性的选择性渗透膜. 近年来, 石墨烯薄膜在过滤、分离和海水淡化等领域 展现出潜在的应用价值.

同时, 石墨烯的二维单原子结构使其在吸附材 料方面具有巨大的优势, 高比表面积使其有效吸附 表面远高于碳纳米管(carbon nanotubes，CNTs). 另 外, 同传统吸附剂相比, 石墨烯及其复合材料的多孔 结构使其具有更高的吸附速率，能够快速吸附各种 污染物, 如染料、重金属和有机物等, 是一种比较理 想的吸附材料 ${ }^{[6]}$. 本文综述了石墨烯薄膜的传质现象 和石墨烯基纳米材料对污染物的吸附, 展望了石墨 烯材料在膜分离、脱盐、污染物去除等水处理领域的 潜在应用。

\section{1 石墨烯薄膜的传质现象}

\section{1 纳米孔石墨烯薄膜的传质现象}

通过在单层石墨烯上引人纳米孔, 可获得一种 有效的分离膜: 纳米孔石墨烯薄膜 (nanoporous graphene, NG). NG膜的化学、力学性能稳定, 具有超薄 的厚度. 单层石墨烯对最小的 $\mathrm{He}$ 气分子也具有很好 的阻隔性 ${ }^{[7]}$. 因此, 最初的理论研究侧重于在石墨烯 上进行打孔，并对其进行功能化，以实现对水、气体 和离子的选择性渗透. 
$\mathrm{Du}$ 等人 ${ }^{[8]}$ 设计了一系列不同尺寸和形状的多孔 石墨烯薄膜, 如图 1(a)所示, 根据气体渗透机理的不 同来分离 $\mathrm{H}_{2} / \mathrm{N}_{2}$. Sint等人 ${ }^{[9]}$ 在石墨烯纳米片上设计了 不同功能化的纳米孔, 如图1(b)所示, 通过分子动力 学模拟发现石墨烯纳米片具有高离子选择性, 是一 种很好的离子篮材料. Cohen-Tanugi和Grossman ${ }^{[10]}$ 也 利用传统分子动力学模型模拟了功能化的单层石墨 烯, 发现其能够使水分子畅通无阻通过, 而有效阻隔 $\mathrm{NaCl}$ 盐离子, 如图 1(c)所示. 同时, 渗透的水流速比 传统反渗透膜高几个数量级. 对不同孔隙直径和孔 隙边缘不同功能化的多孔石墨烯的模拟结果表明, 脱盐性能和孔径大小及功能化程度密切相关. 在石 墨烯薄膜上引人具有识别效应的纳米孔, 可有效辨 别 $\mathrm{Na}^{+}$和 $\mathrm{K}^{+[11]}$. 上述研究结果表明了多孔石墨烯用于 海水淡化的可行性.

上述理论研究结果被后续一系列实验研究证实. Koenig等人 ${ }^{[12]}$ 利用紫外线诱导氧化刻蚀方法在毫米 尺寸的石墨烯薄膜上引人亚微米级孔隙, 分别对 $\mathrm{H}_{2}$, $\mathrm{CO}_{2}, \mathrm{Ar}, \mathrm{N}_{2}, \mathrm{CH}_{4}$ 和 $\mathrm{SF}_{6}$ 几种气体进行加压渗透测试.

(a)

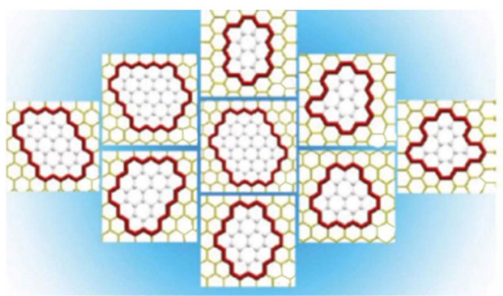

(b)

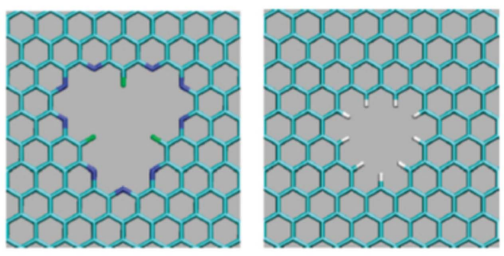

(c)

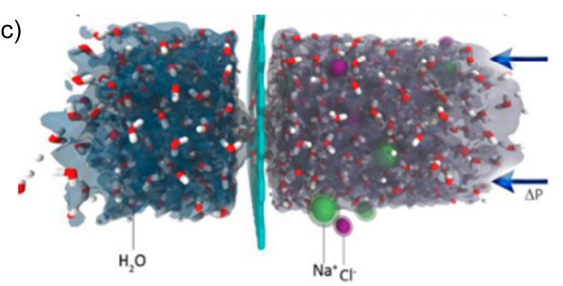

图 1 (网络版彩色)(a) 多孔石墨烯结构模型 ${ }^{[8]}$; (b) 功能化的石墨烯 纳米孔: (左) 氮和氟刻蚀形成的 F-N 纳米孔; (右) 氢刻蚀形成的 $\mathrm{H}$ 纳 米孔 ${ }^{[9]}$; (c) 计算模拟侧面示意图 ${ }^{[10]}$

Figure 1 (Color online) (a) Structure of porous graphene models ${ }^{[8]}$; (b) functionalized graphene nanopores: F-N-terminated nanopore (left), H-terminated nanopore ${ }^{[9]}$ (right); (c) side view of the computational system $^{[10]}$
结果表明, 石墨烯多孔薄膜可以实现气体的有效分 离. Celebi等人 ${ }^{[13]}$ 通过物理穿孔方法在独立存在的双 层石墨烯上制备出数百万紧密相连、孔径分布在 $<10$ $\mathrm{nm}$ 至 $1 \mu \mathrm{m}$ 之间的纳米孔. 利用不同孔径的 NG对气 体、液体和水蒸气的传输进行测试, 发现其可以分离 不同气体, 且由于其原子尺寸厚度、传输速率远高于 目前现有的有限厚度二维薄膜. O'Hern等人 ${ }^{[14]}$ 利用 化学气相沉淀法(chemical vapor deposition, CVD)在 多孔基底上制备出尺寸大于 $25 \mathrm{~mm}^{2}$ 的单层石墨烯薄 膜, 由于石墨烯薄膜上具有孔径分布在 1 15 nm的缺 陷, 所以具有分子选择性. 之后, 他们又利用离子溅 射和氧化刻蚀方法在石墨烯晶格上引人宏观可控、高 密度 $\left(10^{12} \mathrm{~cm}^{-2}\right)$ 、亚纳米直径 $(0.40 \pm 0.24 \mathrm{~nm})$ 的孔隙, 且使石墨烯的结构能够保持完整. 在实验过程中, 通 过调控刻蚀时间来控制 $\mathrm{NG}$ 的选择性能，当氧化时间 较短时, 由于阴离子和孔隙边缘带负电的官能团发 生静电排斥作用, 因此孔隙具有阳离子选择性; 当氧 化时间较长时, 孔隙允许盐离子通过, 但是由于孔径 的尺寸选择性, 却可阻止有机物分子通过 ${ }^{[15]}$. Surwade等人 ${ }^{[16]}$ 利用等离子体刻蚀的方法在单层的石墨 烯上引人纳米孔, 对盐离子的去除率高达 $100 \%, 40^{\circ} \mathrm{C}$ 时水通量高达 $10^{6} \mathrm{~g} /(\mathrm{m} \mathrm{s})$.

综上所述, 在理论上, 可通过控制石墨烯上纳米 孔的尺寸、形状和不同功能化来控制石墨烯的选择分 离性能. $\mathrm{NG}$ 在气体分离、海水淡化等水处理领域具有 诱人的应用前景. 但是, 在实际应用中, $\mathrm{NG}$ 不仅需要 具有较高的强度, 还需要精确的打孔技术. 目前多采 用CVD法合成大面积的石墨烯 ${ }^{[17]}$, 但此类石墨烯产 物大多为多晶结构, 强度较差, 不适合应用于高压状 态. 另外, 传统的打孔技术仍存在诸多不足, 不仅孔 径尺寸不均匀, 且孔径过于集中某一区间，进一步削 弱了 $\mathrm{NG}$ 的强度. 因此, 目前 NG大多仍处于理论研究 阶段, 实验研究较少, 应用于实际尚需要进一步的 研究.

\section{2 氧化石墨烯薄膜的传质现象}

氧化石墨烯 (graphene oxide, GO)是石墨烯的一 种衍生物, 通常由改进的Hummers法制得 ${ }^{[18]}$, 其结 构可以看作是石墨烯的表面和边缘镶嵌着大量的含 氧官能团(如羟基、羧基、环氧基和羰基等 ${ }^{[19]}$. 该结 构和水通蛋白的结构类似, 由于蛋白质具有很好的 离子识别功能, 类比可推断GO在过滤、分离和仿生 
离子传输可能具有潜在的应用. GO微片通过超声处 理, 能够很好地分散在水中, 通过旋涂 ${ }^{[20,21]}$ 、滴 凃 ${ }^{[22,23]}$ 和真空抽滤 ${ }^{[24,25]]}$ 等传统成膜方法处理后, GO 微片可堆叠成肉眼可见的层状薄膜. 这种薄膜的层 片之间可形成具有选择性的二维纳米通道. 另外, $\mathrm{GO}$ 层片间由于层间存在较强的氢键, 所以具有很好 的力学性能, 能够脱离基底而独立存在. 由于上述的 结构特点, GO层状薄膜的气体、水分子和溶液中离子 的传质现象被广泛研究.

\subsection{1 气体和水分子的传输}

首先, 关于 $\mathrm{GO}$ 薄膜对气体和水分子的传质现象 被大量研究. Nair等人 ${ }^{[26]}$ 率先研究了 GO薄膜的传质 现象, 如图2所示. 采用旋涂方法制成亚微米厚度的 GO薄膜, 如图2(a) (c) 所示. 一系列实验结果表明, $\mathrm{GO}$ 薄膜对液体、蒸汽及气体(包括氦气) 完全不透, 如 图2(d)和(e)所示. 但是该薄膜可使水分子畅通无阻碍 通过(图2(f)). 基于以上研究, Boukhvalov等人 ${ }^{[27]}$ 根据 第一性原理密度泛函理论, 建立了水和GO混合系统 的原子模型来探究水的异常渗透现象, 发现在 GO层 片之间形成的六方冰双层及其在层片边缘的融化转 变对水穿过整个 GO堆垛结构至关重要, 从而实现了 优异的水通透性. 通过对 GO进行还原处理或施加压
力来控制 GO层片间距，可对水流速进行控制. 对少 数层状 GO微片(厚度为 $3 \sim 10 \mathrm{~nm}$ )进行组装, 通过不同 的堆垛方法控制薄膜中的通道可实现对气体的选择 性. 在较高相对湿度下, 连接较好的 $\mathrm{GO}$ 薄膜可以实 现高效 $\mathrm{CO}_{2} / \mathrm{N}_{2}$ 分离 ${ }^{[28]}$. $\mathrm{Li}$ 等人 ${ }^{[29]}$ 利用真空抽滤法制 备了厚度约 $1.8 \mathrm{~nm}$ 的超薄 $\mathrm{GO}$ 薄膜, 具有一定的结构 缺陷, 对 $\mathrm{H}_{2} / \mathrm{CO}_{2}$ 及 $\mathrm{H}_{2} / \mathrm{N}_{2}$ 混合气体可分别实现高达 3400 和 900 的选择性, 比最先进的微孔滤膜高出 1 2 个数量级. 利用超薄 $\mathrm{GO}$ 膜从混合气体中分离出 $\mathrm{H}_{2}$ 将 会有很好的应用前景.

以上研究结果表明, GO薄膜对气体具有选择 性 $^{[28,29]}$, 这看似同 Nair等人 ${ }^{[26]}$ 的结果矛盾, 其实不 然. 由于前者制备的 $\mathrm{GO}$ 薄膜皆为数纳米厚、较薄, 连续的纳米毛细管网络尚未形成, 气体可通过缺陷 进行渗透; 而后者的 $\mathrm{GO}$ 薄膜为微米厚度, 具有连续 的 GO毛细管通道网络, 缺陷几乎可以忽略, 所以气 体无法透过.

以上研究都是针对气体和水蒸气的渗透性能研 究, 关于液态水的渗透现象要比气体复杂得多, 这是 因为被 $\mathrm{GO}$ 薄膜所分离的源液和滤液之间，在宏观和 微观尺度上都没有可分辨的不同点. Sun等人 ${ }^{[30]}$ 利用 元素追踪方法解决了这一问题, 在无压力状态下研 (a)

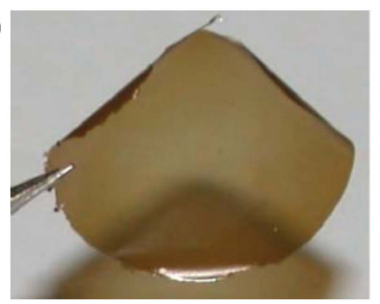

(d)

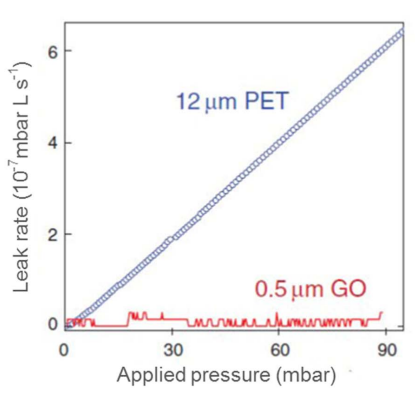

(b)

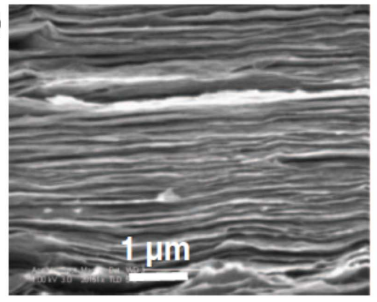

(e)

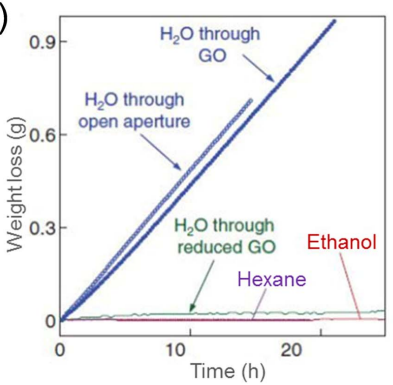

(c)

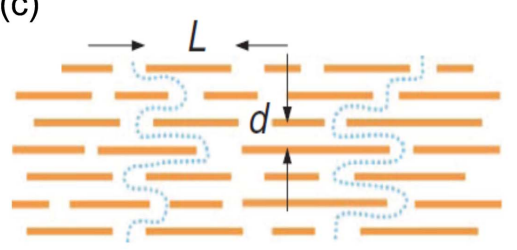

(f)

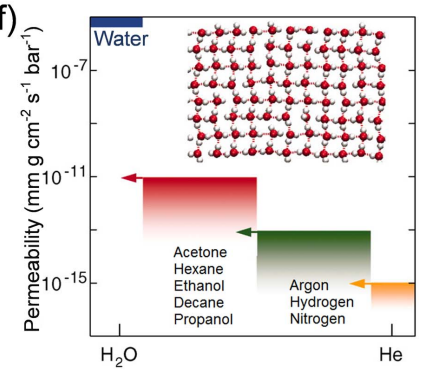

图 2 (网络版彩色)(a) 从铜䈃上剥落的 $1 \mu \mathrm{m}$ 厚的 $\mathrm{GO}$ 薄膜; (b) GO 薄膜的侧面电子显微图; (c) 水分子渗透示意图; (d) PET 膜和独立存在的亚 微米厚度 $\mathrm{GO}$ 薄膜对 $\mathrm{He}$ 泄漏测量对比实验 $\left(1 \mathrm{mbar}=100 \mathrm{~Pa}\right.$ ); (e) 室温下密闭容器中 $\mathrm{H}_{2} \mathrm{O}$ 和乙醇、乙烷的质量随时间的变化; (f) $\mathrm{H}_{2} \mathrm{O}$ 和一些小 分子透过 $\mathrm{GO}$ 薄膜速率的对比 ${ }^{[2]}$

Figure 2 (Color online) (a) Photograph of a $1 \mu \mathrm{m}$ thick GO film peeled off from a Cu foil; (b) electron micrograph of the film's cross section; (c) schematic view for possible permeation through the laminates; (d) examples of He-leak measurements for a freestanding submicron-thick GO membrane and a reference PET film (1 mbar=100 Pa); (e) weight loss for a container sealed with a GO film; (f) permeability of GO paper with respect to water and various small molecules ${ }^{[26]}$ 
究了液态水透过在微孔滤膜基底上 $G O$ 薄膜 (GOCM) 的性能. 采用一定量的重水 (氧化気, $\mathrm{D}_{2} \mathrm{O}$ ) 作为示踪 物来标记液态水, 根据其渗透特性来研究液态水的 渗透特性. 在源液中加人 $\mathrm{MgCl}_{2}$, 分别对源液和滤液 中的水进行追踪, 如图3(a)和(b)所示, 发现离子的渗 透系数和水的渗透系数在同一个数量级, 如图3(c) 所 示. 渗透系数比孔径小于微米级的聚合通道高4 5个 数量级, 且离子的传输主要依赖于水流, 离子和纳米 毛细管的轻微接触对离子的快速传输产生一定的影 响, 如图3(d)所示. 这一现象表明 GO薄膜具有半渗 透性, 同时水分子在通过 $\mathrm{GO}$ 薄膜时倾向于逆离子梯 度方向传输.

上述研究阐明了水分子能够高速透过 $\mathrm{GO}$ 膜的机 理, 且超薄的 $\mathrm{GO}$ 膜对气体有选择性. 接下来将着重 介绍 $\mathrm{GO}$ 薄膜对溶液中的离子的渗透行为.

\subsection{2 溶液中离子的传输}

上述研究针对的是 $\mathrm{GO}$ 薄膜对气体和水分子的传 质现象, 基于当前严重的淡水危机, $\mathrm{GO}$ 薄膜在海水 淡化领域的应用主要是去除海水中的盐离子, 探究 $\mathrm{GO}$ 薄膜的离子传质行为具有更为重要的实用意义.

Sun等人 ${ }^{[31]}$ 采用滴涂法制备了微米级厚度的独 立存在的 GO薄膜, 研究了溶液中不同离子的渗透行
为, 如图 4(a)和(b)所示. 在 GO膜的两端分别加人等 体积的盐溶液和去离子水, 发现钠盐很快发生渗透, 而重金属盐较慢，铜盐和有机物被 GO有效阻挡. 这 一结果表明, 可以从铜盐和有机物中有效分离出钠 盐. 进一步对离子透过 GO膜的机理进行了研究, 对 水合半径顺序为 $\mathrm{Mn}^{2+}>\mathrm{Cd}^{2+}>\mathrm{Cu}^{2+}>\mathrm{Na}^{+}$的几种盐进行 测试, 渗透结果为 $\mathrm{Na}^{+}>\mathrm{Mn}^{2+}>\mathrm{Cd}^{2+}>\mathrm{Cu}^{2+}$. 这说明离子 渗透同其水合半径的大小没有直接关系，而是由于 金属盐离子和 $\mathrm{GO}$ 片上的官能团的不同相互作用造成 的. 对相同浓度的几种钠盐溶液的渗透进行测试，渗 透结果为 $\mathrm{NaOH}>\mathrm{NaHSO}_{4}>\mathrm{NaCl}>\mathrm{NaHCO}_{3}$, 说明盐溶 液中的阴离子也会对渗透结果产生影响. 随后, 研究 了不同盐离子的渗透机理 ${ }^{[32,33]}$, 如图 4(c) 和(d) 所示. 结果表明，过渡金属阳离子倾向于和 $\mathrm{GO}$ 片的含氧官 能团相互作用，主族金属阳离子更倾向于通过阳离 子 $-\pi$ 键和 $\mathrm{sp}^{2}$ 基团相互作用，离子的不同相互作用导 致 $\mathrm{GO}$ 膜具有不同的离子选择能力. 当 $\mathrm{GO}$ 薄膜被氨 基酸目标官能团修饰时，离子的渗透性能减弱. 这说 明可以在 $\mathrm{GO}$ 膜上修饰目标官能团来调制离子的运输 速率. 上述研究不仅阐明了 $\mathrm{GO}$ 薄膜的选择性离子渗 透机理，也为在 $\mathrm{GO}$ 薄膜上修饰目标官能团来分离目 标离子的研究奠定了基础. (a)

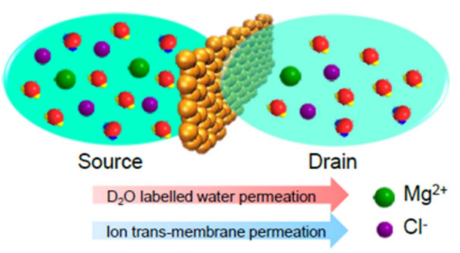

(c)

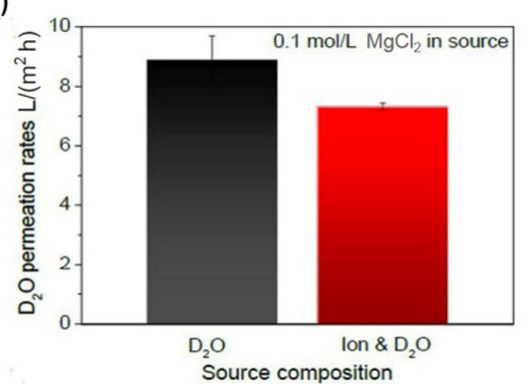

(b)

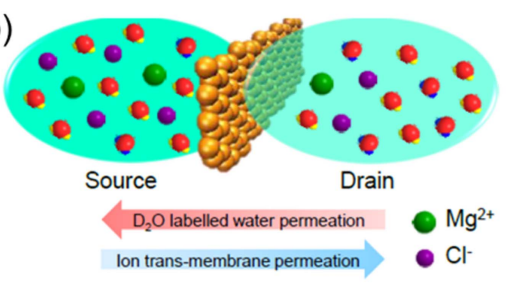

(d)

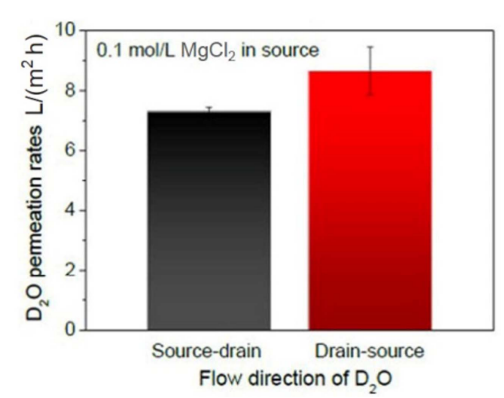

图 3 (网络版彩色) 源液中为 $0.1 \mathrm{~mol} / \mathrm{L} \mathrm{MgCl}_{2}$ 时 $\mathrm{D}_{2} \mathrm{O}$ 对水渗透追踪 ${ }^{300}$. (a) 采用 $30 \%$ (质量百分比) 的 $\mathrm{D}_{2} \mathrm{O}$ 对源液中 $\mathrm{H}_{2} \mathrm{O}$ 进行追踪; (b) 源液为 $0.1 \mathrm{~mol} / \mathrm{L}$ 的 $\mathrm{MgCl}_{2}$, 漏夜采用 $30 \%$ (质量百分比)的 $\mathrm{D}_{2} \mathrm{O}$ 进行标记; (c) 源液中含和不含 $\mathrm{MgCl}_{2}$ 的 $\mathrm{H}_{2} \mathrm{O}$ 透过 $\mathrm{GOCM}$ 的渗透对比; (d) 源液中含 $\mathrm{MgCl}_{2}$ 时 $\mathrm{H}_{2} \mathrm{O}$ 从 2 个方向透过率的对比

Figure 3 (Color online) $\mathrm{D}_{2} \mathrm{O}$ labelled water permeation in the presence of $0.1 \mathrm{~mol} / \mathrm{L} \mathrm{MgCl}_{2}$ in sources ${ }^{[30]}$. (a) Schematic drawing for the labelling of $0.1 \mathrm{~mol} / \mathrm{L} \mathrm{MgCl}_{2}$ source solution by $30 \mathrm{wt} \% \mathrm{D}_{2} \mathrm{O}$ tracers; (b) schematic diagram for the labelling of drain solutions by 30 wt\% $\mathrm{D}_{2} \mathrm{O}$ tracers when dissolving $0.1 \mathrm{~mol} / \mathrm{L} \mathrm{MgCl}_{2}$ in source solutions; (c) water permeations through GOCM membranes in both directions when dissolving ions in sources; (d) water permeation rates through GOCM membranes in both directions in the presence of source ions 
(a)

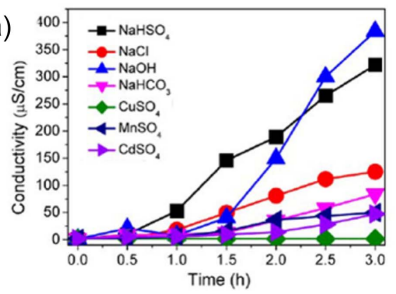

(b)

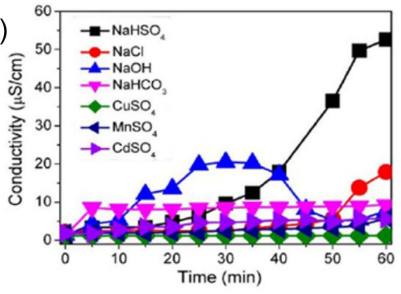

(c)

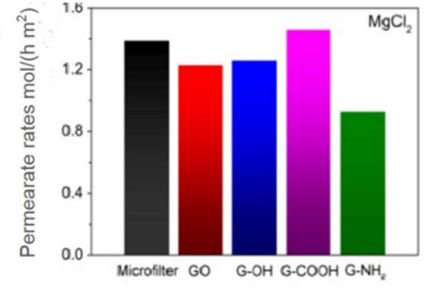

(d)

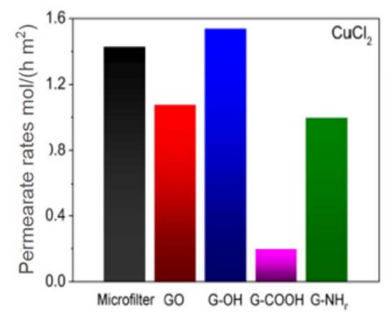

(e)

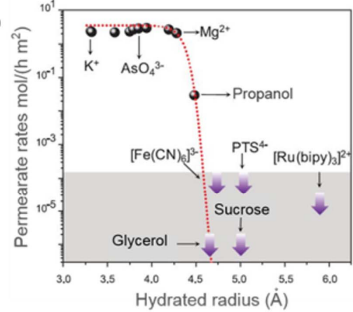

图 4 (网络版彩色)(a), (b) 不同盐离子透过 GO 薄膜随时间变化趋势 ${ }^{[31]} ;$ (c), (d) $\mathrm{Mg}^{2+}, \mathrm{Cu}^{2+}$ 透过 $\mathrm{GO}$ 和不同功能化的石墨烯薄膜对比 ${ }^{[32]}$; (e) 不 同离子的水合半径大小透过 $5 \mu \mathrm{m}$ 厚的 $\mathrm{GO}$ 薄膜渗透速率比较 ${ }^{[34]}$

Figure 4 (Color online) (a) Penetration processes of different ionic compounds through GO membranes; (b) initial stages of the penetration process$\mathrm{es}^{[31]}$; (c) $\mathrm{Cu}^{2+}$ and (d) $\mathrm{Mg}^{2+}$ permeation rates through blank microfilters, GO, G-OH, G-COOH, and G-NH $\mathrm{H}_{2}$ membranes with the cellulose microfilters underneath ${ }^{[32]}$; (e) sieving through the atomic-scale mesh $^{[34]}$

Joshi等人 ${ }^{[34]}$ 通过真空抽滤 GO分散液制备了微 米厚度的薄膜分子篮. 对不同半径的离子进行实验 结果表明, GO膜能够阻挡所有水合半径大于 $0.45 \mathrm{~nm}$ 的离子, 如图 $4(\mathrm{e})$ 所示. 半径小于 $0.45 \mathrm{~nm}$ 的离子渗透 速率比自由扩散高出数千倍. GO薄膜的这一特性在 膜分离领域具有非常重要的应用价值.

$\mathrm{Mi}^{[35]}$ 提出, 通过调控 $\mathrm{GO}$ 层片间距可以从目标溶 液中分离出目标离子, 如图 5所示. 例如, 对于脱盐 应用, 需要将 $\mathrm{GO}$ 的层间距减小至 $0.7 \mathrm{~nm}$ 以下 $\left(\mathrm{Na}^{+}\right.$水 合直径为 $0.72 \mathrm{~nm}$ ), 可通过部分还原 $\mathrm{GO}$ 或者在 $\mathrm{GO}$ 层 片间插人二维的纳米薄片来实现. 与此相反, 如果要 扩大GO的层间距至1 2 $\mathrm{nm}$, 可在 $\mathrm{GO}$ 层片间插人刚 性较大的化学基团或聚合物链. 所获得的结构可以 应用于水净化、废水处理、药物和燃料分离. 若使 $\mathrm{GO}$ 膜层间距进一步增大到 $2 \mathrm{~nm}$, 则可以在 $\mathrm{GO}$ 层片间插 人纳米粒子或纳米纤维, 进一步应用于生物医学的 肾析和透析等领域.

$\mathrm{Hu}$ 和 $\mathrm{Mi}^{[36]}$ 在高分子基底上采用交联剂将 $\mathrm{GO}$ 纳 米片沉积成叠层 GO薄膜, 如图6(a)所示. 这种堆叠 的 GO薄膜在水溶液中较稳定, 测试发现 GO薄膜的 水流速在80 276 L m m $^{-2} \mathrm{~h}^{-1} \mathrm{MPa}$, 比商业纳滤膜高 $4 \sim 10$ 倍. 对单价和二价盐的脱盐率为 6\% 46\%, 对罗 丹明 $\mathrm{B}(\mathrm{RhB})$ 的去除率为 46\% 66\%, 对亚甲基蓝( MB) 的去除率高达93\% 95\%, 如图6(b)和(c)所示. 另有研 究将化学处理后的石墨烯分散在微孔滤膜基底上,
抽滤获得超薄的(22 53 $\mathrm{nm})$ 石墨烯纳滤膜 ${ }^{[37]}$, 其水 透过速率高达 $21.8 \mathrm{~L} \mathrm{~m}^{-2} \mathrm{~h}^{-1} \mathrm{bar}^{-1}$, 对有机染料的去 除率高达 $99 \%$, 脱盐率约为 $20 \%$ 60\%。该渗透膜的脱 盐过程取决于物理篮选和电子相互作用. 最近, Sun 等人 ${ }^{[38]}$ 提出了一种全新的、精确可控的基于 $\mathrm{GO}$ 的复 合渗透膜的设计思路, 将单层二氧化钛 (TO)纳米薄 片插人 GO层状薄膜中, 并辅以微弱的紫外(UV)光照. 在 UV光照射下, TO的高效光催化特性将 GO还原为 $\mathrm{RGO}$, 从而获得 RGO/TO复合渗透膜，如图6(d)所示. 水的渗透速率保持在原来的 $60 \%$, 如图6(e)所示. 另 外, $\mathrm{TO}$ 具有光致亲水特性, 可保证高的水流速率, 在 不施加压力的情况下, $\mathrm{RGO} / \mathrm{TO}$ 复合膜对离子的渗透 同未还原的 GO/TO相比, 降低至 $<5 \%$, 如图6(f)所示. 这种复合薄膜制备方法简单，在水净化领域具有很 好的潜在应用.

完全还原的氧化石墨烯 (RGO)薄膜的脱盐性能 也被广泛研究. $\mathrm{Su}$ 等人 ${ }^{[39]}$ 采用HI对PET基底上的 GO 薄膜进行还原, 得到 $30 \mathrm{~nm}$ 厚的 RGO薄膜, 对 $\mathrm{He}$ 和水 分子完全不能透过. 而厚度超过 $100 \mathrm{~nm}$ 的RGO薄膜 对几乎所有气体、液体和腐蚀性化学试剂(如HF)都不 具有透过性. 与此结果相反, $\mathrm{Liu}$ 等人 ${ }^{[40]}$ 最近报道, 通 过真空抽滤法在微孔滤膜基底上制备几十到几百纳 米厚的 $\mathrm{GO}$ 薄膜, 采用 $\mathrm{HI}$ 蒸气进行还原后能够独立存 在. 对 $\mathrm{NaCl}$ 溶液进行测试, 这种 $\mathrm{RGO}$ 膜不仅具有高 水通透速率, 且和商业膜相比, GO膜具有更高的脱 
(a)

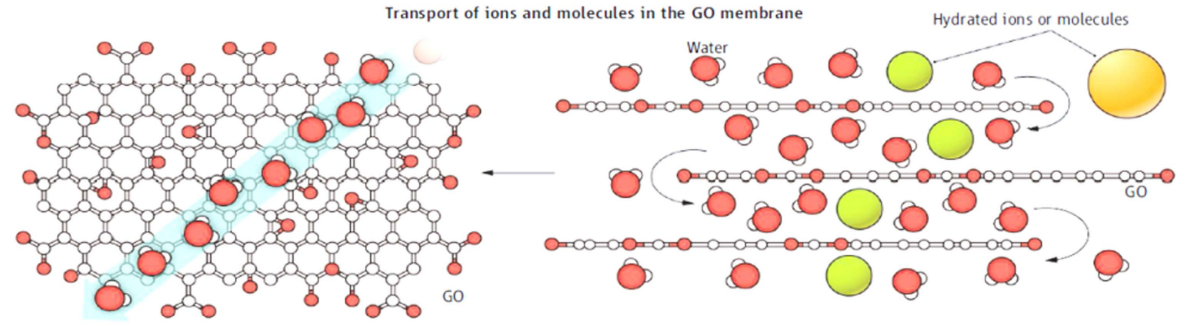

(b)

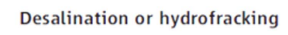

Water, fuel, or chemical purification

Biomedical filtration

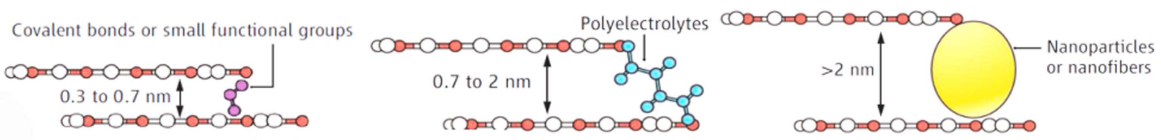

(c)

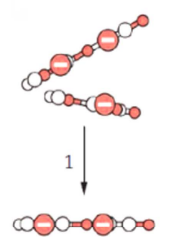

Vacuum filtration

Layer-by-layer assembly
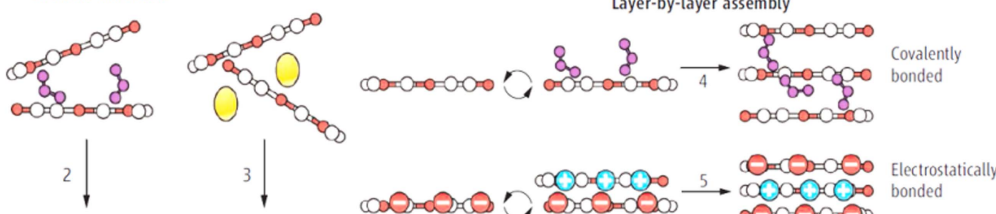

$\mathrm{cos}+\mathrm{cos}=0$

$\mathrm{CO}=0 \mathrm{O}=0$

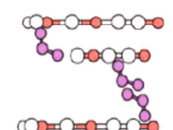

$\cos =0=0=0=0$

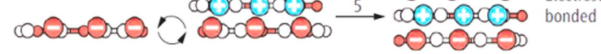

$C O D=0=0=0=0$

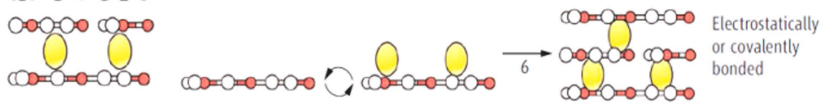

图 5 (网络版彩色)氧化石墨烯膜. (a) 水、分子和离子透过层状氧化石墨烯薄膜示意图; (b) GO 薄膜的分离能力与层间距的大小关系; (c) 层状 $\mathrm{GO}$ 薄膜合成的几种设想 ${ }^{[35]}$

Figure 5 (Color online) GO membranes. (a) Water and small-sized ions and molecules (compared with the void spacing between stacked GO nanosheets) permeate superfast in the GO membrane, but largerspecies are blocked; (b) separation capability of the GO membrane is tunable by adjusting the nanochannel size; (c) several methods for the synthesis of GO membranes have been reported or envisioned ${ }^{[35]}$

(a)
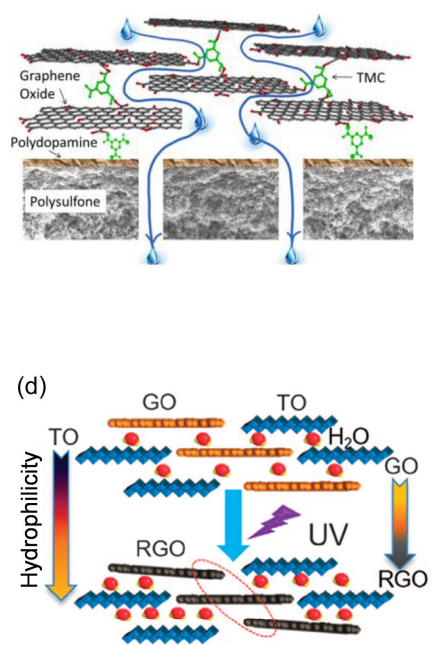

(b)
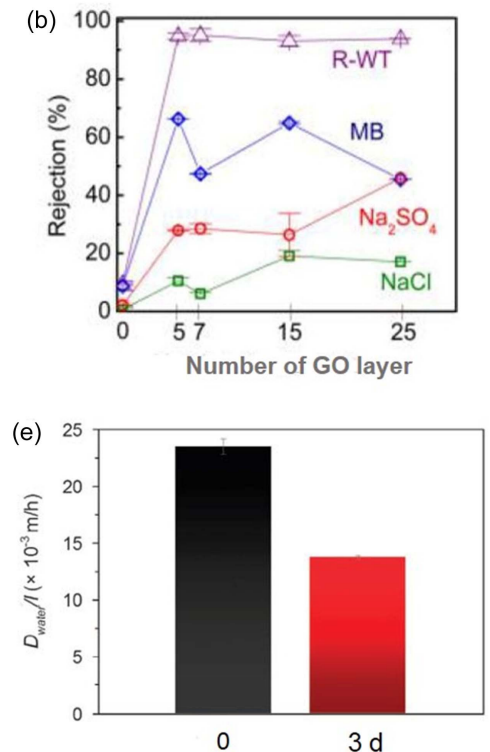
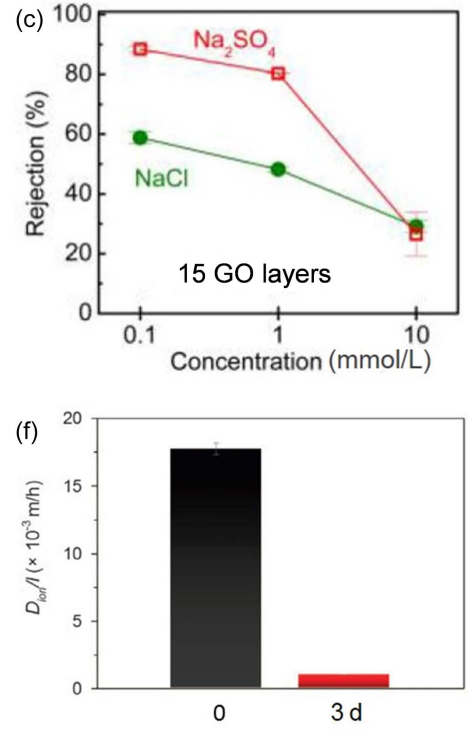

图 6 (网络版彩色)(a) 均苯三甲酰氯(TMC)连接的层状 GO 薄膜结构; (b) 不同层状 GO 薄膜对有机物和盐离子的去除率; (c) 15 层 GO 的脱盐 率随盐浓度的变化 ${ }^{[3]}$; (d) UV 光照下 GO/TO 复合薄膜的还原过程原理示意图; (e) UV 光照 0 和 $3 \mathrm{~d}$ 的 GO/TO 复合薄膜 $\mathrm{D}_{2} \mathrm{O}$ 的扩散系数; (f) UV 光照 0 和 $3 \mathrm{~d}$ 的 $\mathrm{GO} / \mathrm{TO}$ 复合薄膜离子扩散系数对比 ${ }^{[38]}$

Figure 6 (Color online) (a) Layer GO membrane structure with TMC connection; (b) rejection of salts and organic dyes with different numbers of $\mathrm{GO}$ (c) layers; effect of salt concentration on the rejection by the 15-layered GO membrane ${ }^{[36]}$; (d) schematic diagram for the reduction of GO by TO within the hybrid membranes under UV irradiation; (e) $\mathrm{D}_{2} \mathrm{O}$ diffusion coefficients through GO/TO membranes after UV irradiation for 0 and $3 \mathrm{~d}$, respectively; (f) ion diffusion coefficients through GO/TO membranes after UV irradiation for 0 and $3 \mathrm{~d}$, respectively ${ }^{[38]}$ 
盐性能. 出现上述截然不同的结果的可能原因是, 仅 通过肉眼观察颜色并不能确保GO被完全还原, GO很 可能是部分被还原, 还有可能是在操作过程中破坏 了 GO的微观结构, 尚需进一步研究确认.

\section{2 石墨烯纳米材料对污染物的吸附}

\section{1 对重金属离子的吸附}

工业的快速发展已经造成了重金属离子污染, 这些污染严重危害着人类的健康. 目前, 使用纳米材 料吸附重金属离子已经逐渐引起人们的关注. 而石 墨烯材料由于可控的孔结构和高比表面积对重金属 离子具有良好的吸附性能.

Huang等人 ${ }^{[41]}$ 采用真空低温剥离的方法制备了 石墨烯纳米片 (graphene nanosheets, GNSs), 对水溶 液中 $\mathrm{Pb}^{2+}$ 最大的吸附量可达 $35 \mathrm{mg} / \mathrm{g}$. 将 $\mathrm{GO}$ 还原为 RGO也可以吸附水中的重金属, Leng等人 ${ }^{[42]}$, 采用水 合肼还原的 $\mathrm{GO}$ 对 $\mathrm{Sb}^{3+}$ 的最大吸附量为 $7.4 \mathrm{mg} / \mathrm{g}$, 经过 5 次吸附/脱附循环后，吸附率为原来的 $60 \%$.

上述结果表明, 石墨烯虽然具有良好的吸附金 属离子性能, 但是由于表面没有活性基团, 仅能通过 范德华力或疏水相互作用吸附重金属离子. 而GO作 为石墨烯的一种衍生物, 表面含有大量的含氧官能 团, 在水中带负电, 由于大多数的重金属离子是阳离 子性, 容易吸附在 GO吸附剂上形成络合物, 更有利 于吸附水中的的重金属.

Yang等人 ${ }^{[43]}$ 研究了 $\mathrm{GO}$ 对 $\mathrm{Cu}^{2+}$ 的吸附情况, 由于 $\mathrm{GO}$ 和 $\mathrm{Cu}^{2+}$ 的相互作用, GO片发生褶皱形成大的聚合 物, 在浓度和吸附平衡时估算了 $\mathrm{GO}$ 的最大吸附量, 约为活性炭的 10 倍. Wang 研究组 ${ }^{[44,45]}$ 研究了 GO对 $\mathrm{Zn}^{2+}$ 和 $\mathrm{U}^{6+}$ 吸附, 最大吸附量分别达到 246 和 $299 \mathrm{mg} / \mathrm{g}$. Zhao 等人 ${ }^{[46]}$ 随后针对放射性元素 $\mathrm{U}^{6+}$ 研究了离子浓 度、温度和 $\mathrm{pH}$ 对吸附的影响, 发现 $\mathrm{pH}$ 对 GO的吸附具 有强烈的影响, 而离子强度对其吸附几乎没有影响. 这说明 $\mathrm{GO}$ 的吸附主要是其内部表面的络合而不是外 表面的络合和离子交换. 当 $\mathrm{pH}$ 为 $5.0 \pm 0.1$ 、温度为 $20^{\circ} \mathrm{C}$ 时, $G O$ 对 $\mathrm{U}^{6+}$ 的吸附量为 $97.5 \mathrm{mg} / \mathrm{g}$, 优于之前报道的 纳米材料的吸附性能.

除了对单一离子的吸附研究, 另有研究采用 GO 来吸附不同种类的离子, 进一步考查了 $\mathrm{GO}$ 对不同金 属离子的吸附能力. Zhao等人 ${ }^{[47]}$ 制备了层状 $\mathrm{GO}$ 纳米 片并对水溶液中的 $\mathrm{Cd}^{2+}$ 和 $\mathrm{Co}^{2+}$ 的吸附进行了研究, 探
测了 $\mathrm{pH}$ 、离子强度和腐植酸对其吸附的影响. 结果表 明, $\mathrm{pH}$ 值对 GO的吸附具有强烈的影响, 而离子强度 对其吸附影响很小, 当 $\mathrm{pH}<8$ 时, 腐植酸降低了 $\mathrm{GO}$ 的 吸附性能. 当 $\mathrm{pH}$ 为 $6.0 \pm 0.1$ 、温度为 $303 \mathrm{~K}$ 时, 对 $\mathrm{Cd}^{2+}$ 和 $\mathrm{Co}^{2+}$ 吸附量分别高达 106.3 和 $68.2 \mathrm{mg} / \mathrm{g}$. Liu等人 ${ }^{[48]}$ 研究了 $\mathrm{GO}$ 对 $\mathrm{Au}^{3+}, \mathrm{Pd}^{2+}$ 和 $\mathrm{Pt}^{4+}$ 的吸附性能, 探索了 $\mathrm{pH}$ 、初始浓度、接触时间和温度对吸附的影响, 发现 $\mathrm{pH}$ 为 6.0 时, 吸附效果最好, 对 $\mathrm{Au}^{3+}, \mathrm{Pd}^{2+}$ 和 $\mathrm{Pt}^{4+}$ 的最 大吸附量分别为 $108.3,80.7$ 和 $71.3 \mathrm{mg} / \mathrm{g}$, 吸附等温线 符合Langmuir方程. Stiko等人 ${ }^{[49]}$ 研究了 GO同时吸附 多种金属离子, 对 $\mathrm{Cu}^{2+}, \mathrm{Zn}^{2+}, \mathrm{Cd}^{2+}$ 和 $\mathrm{Pb}^{2+}$ 的吸附, 当 $\mathrm{pH}=5$ 时, 吸附量分别为 $294,345,530,1119 \mathrm{mg} / \mathrm{g}$. 竞 争吸附实验结果表明, 亲和力顺序为 $\mathrm{Pb}^{2+}>\mathrm{Cu}^{2+}>$ $\mathrm{Cd}^{2+}>\mathrm{Zn}^{2+}$. 对吸附后的样品进行观察, 发现有结块 和沉淀的倾向，如图7(a) (c) 所示. GO 良好的分散性 及其与金属相互作用形成沉淀是从水中去除金属离 子的主要途径, 目前, $\mathrm{GO}$ 在分析化学中作为固体吸 附剂吸附微量元素和重金属离子的优异能力远远高 于现有的其他吸附剂.

磁性石墨烯复合材料在水处理中具有易于分离、 环境友好等优点而引起人们的关注. Zhang等人 ${ }^{[50]}$ 制 备了磁性铁钴氧化物 $\left(\mathrm{CoFe}_{2} \mathrm{O}_{4}\right)-\mathrm{RGO}$ 复合材料, 用于 去除水溶液中的 $\mathrm{Pb}^{2+}$ 和 $\mathrm{Hg}^{2+}$, 吸附量分别高达 299.4 和 $157.9 \mathrm{mg} / \mathrm{g}$. Liu等人 ${ }^{[51]}$ 通过共沉淀法制备了 $\mathrm{Fe}_{3} \mathrm{O}_{4} / \mathrm{GO}$ 磁性纳米复合材料来去除水中的 $\mathrm{Co}^{2+}$, 并 比较了 $\mathrm{Fe}_{3} \mathrm{O}_{4} / \mathrm{GO}$ 复合材料和 $\mathrm{Fe}_{3} \mathrm{O}_{4}$ 的吸附性能, 对 $\mathrm{Co}^{2+}$ 的最大吸附量分别为 12.98 和 $6.2 \mathrm{mg} / \mathrm{g}$. Chen等 人 ${ }^{[52]}$ 用四方纤铁矿修饰的 $\mathrm{GO}$ 来吸附 $\mathrm{As}^{3+}$ 和 $\mathrm{As}^{5+}$, 吸 附量高达 77.5 和 $45.7 \mathrm{mg} / \mathrm{g}, \mathrm{As}^{3+}$ 和 $\mathrm{As}^{5+}$ 的浓度为 100 $\mu \mathrm{g} / \mathrm{L}$ 时, 经过5次循环后去除率仍高达 $100 \%$ 和 $97 \%$.

通过对 GO进行改性或复合, 可进一步增强吸附 效果. Madadrang等人 ${ }^{[53]}$ 采用乙二胺四乙酸(EDTA)来 改性 GO, 由于EDTA能与金属形成稳定的螯合剂, 如图7(d)和(e)所示, 所以具有良好的吸附性能. 石墨 烯复合材料在10 30 min达到吸附平衡, 速度高于其 他碳基吸附材料. 金属离子在 $\mathrm{GO}$ 表面的解析行为表 明, 采用 $\mathrm{HCl}$ 处理后 $\mathrm{GO}$ 可以重复利用, 对 $\mathrm{Pb}^{2+}$ 的最大 吸附量为 $479 \pm 46 \mathrm{mg} / \mathrm{g}$ (图7(f)), 比 GO的吸附量高1 2 倍, 比碳纳米管的吸附量高4 5倍, 表明EDTA-GO在 去除重金属离子方面是一种很好的吸附剂. 最近, Dong等人 ${ }^{[54]}$ 制备了 GO和实施矿物的纳米复合材料 $(\mathrm{GO}-\mathrm{SCH})$ 来吸附水中的 $\mathrm{Sb}^{5+}$, 在 $\mathrm{pH} 7, \mathrm{Sb}^{5+}$ 的浓度 
(a)

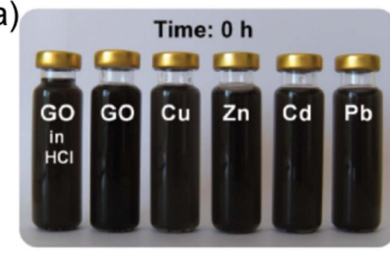

(d)

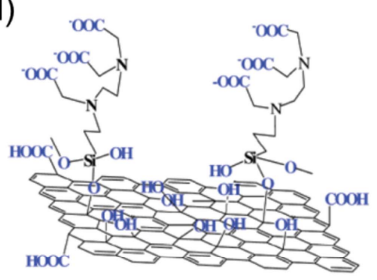

(b)

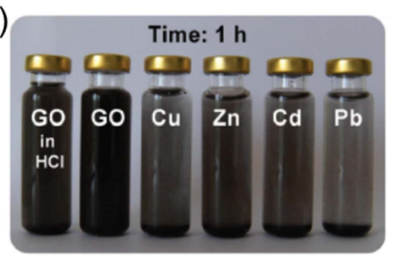

(e)

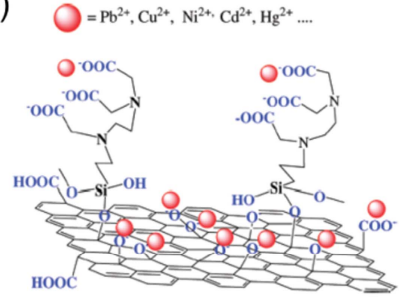

(c)

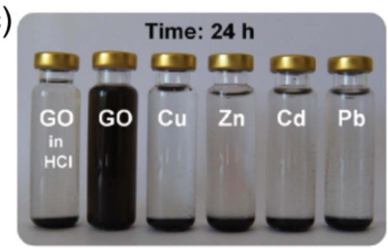

(f)

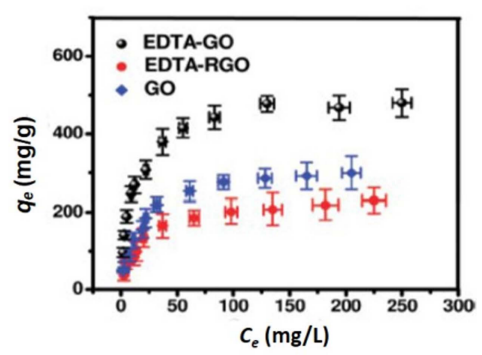

图 7 (网络版彩色)(a) (c) GO 吸附重金属离子的聚集和沉淀 ${ }^{[49]}$; (d) EDTA-GO 的化学结构; (e) EDTA-GO 和重金属离子的结合; (f) EDTA-GO, EDTA-RGO, GO 三种吸附剂随 $\mathrm{Pb}^{2+}$ 浓度变化吸附量对比. 实验条件: 起始浓度为 $5 \sim 300 \mathrm{mg} / \mathrm{L}$, 样品剂量为 $25 \mathrm{mg} / 200 \mathrm{~mL}, \mathrm{pH} 6.8$, 温度为 $(25$ 土 $2)^{\circ} \mathrm{C}$, 接触时间 $24 \mathrm{~h} . q_{\mathrm{e}}$ 为吸附剂对金属离子的吸附量 $(\mathrm{mg} / \mathrm{g}), C_{\mathrm{e}}$ 为金属离子在平衡状态下的浓度 $(\mathrm{mg} / \mathrm{L})^{[52]}$

Figure 7 (Color online) (a)-(c) Agglomeration and precipitation of GO with adsorbed metal ions ${ }^{[49]}$; (d) chemical structure of EDTA-GO; (e) interaction between EDTA-GO and heavy metal cations; (f) adsorption isotherms of $\mathrm{Pb}^{2+}$ for EDTA-GO, EDTA-RGO, GO adsorbents. Experiment condition: Initial concentration is $5-300 \mathrm{mg} / \mathrm{L}$, sample dose is $25 \mathrm{mg} / 200 \mathrm{~mL}, \mathrm{pH}=6.8$, temperature is $(25 \pm 2)^{\circ} \mathrm{C}$, contact time is $24 \mathrm{~h}^{[52]} . q_{\mathrm{e}}$ is the adsorption mount of metal ions $(\mathrm{mg} / \mathrm{g}), C_{\mathrm{e}}$ is the equilibrium concentration of metal ions $(\mathrm{mg} / \mathrm{L})^{[52]}$

为 $8.0 \mathrm{mg} / \mathrm{L}$ 时, 吸附量高达 $158.6 \mathrm{mg} / \mathrm{g}$, 吸附性能远 高于 $\mathrm{GO}$ 和 $\mathrm{SCH}$. 由此可见, GO的复合材料能够大大 提高吸附性能, 因此在去除废水中的重金属离子领 域具有潜在的应用价值.

除此之外, 三维石墨烯的吸附也备受关注. 三维 石墨烯不仅易制备、孔隙率高，在保持高吸附量的同 时还能够克服石墨烯和 $\mathrm{GO}$ 等纳米吸附剂易团聚、不 易分离和残余纳米生物毒性等缺点. $\mathrm{Mi}$ 等人 ${ }^{[55]}$ 通过 干燥冷冻方法制备出 $\mathrm{GO}$ 气凝胶, 测试对水溶液中 $\mathrm{Cu}^{2+}$ 的去除性能, 在 $15 \mathrm{~min}$ 内达到吸附平衡, 在室温 下对 $\mathrm{Cu}^{2+}$ 的吸附量约为 $19 \mathrm{mg} / \mathrm{g}$. $\mathrm{Li}$ 等人 ${ }^{[56]}$ 利用 CVD 法以 $\mathrm{NiCl}_{2} \cdot 6 \mathrm{H}_{2} \mathrm{O}$ 为催化剂制备了宏观的三维石墨烯 (3D-GMOs), 作为电解电极去除各种重金属离子. 对 $\mathrm{Cd}^{2+}, \mathrm{Pb}^{2+}, \mathrm{Ni}^{2+}, \mathrm{Cu}^{2+}$ 去除率分别为 $434,882,1.68$ 和 $3.82 \mathrm{mg} / \mathrm{g}$. Lei等人 ${ }^{[57]}$ 合成了独立存在的三维 $G O$ 泡沫 (GOF), 测试对重金属离子如 $\mathrm{Cd}^{2+}, \mathrm{Zn}^{2+}, \mathrm{Pb}^{2+}$ 和 $\mathrm{Fe}^{3+}$ 的吸附性能, 吸附量分别高达252.5, 326.4, 381.3和 $587 \mathrm{mg} / \mathrm{g}$, 远高于传统吸附剂. 在5次循环后, 吸附 性能仍为初始值的 $85 \%$, 表明 GOF也是一种较理想的 吸附剂, 可大范围去除重金属离子.

\section{2 对染料的吸附}

染料是重要的环境污染源之一, 主要来源于印
刷、纺织造纸等行业, 染料具有复杂的芳环结构且具 有生理毒性及致癌作用, 难以生物降解, 主要排放在 废水中, 而废水污染引发的环境问题日益突出. 吸附 法由于效率高、速度快、适应性强和易操作等优点而 被广泛应用于环境污染物治理中. 石墨烯由于高的 比表面积而成为一种优质的吸附剂.

Bradder等人 ${ }^{[58]}$ 首次研究了 $\mathrm{GO}$ 对水中 $\mathrm{MB}$ 和孔雀 石绿的吸附, 吸附量分别为 351 和 $248 \mathrm{mg} / \mathrm{g}$, 吸附能力 远高于石墨和活性炭. 另外, GO片所携带的大量含氧 官能团在吸附过程中发挥了重要的作用, 吸附机理为 静电吸引作用. Yang等人 ${ }^{[59]}$ 研究了 GO对水中MB的吸 附, 吸附能力高达 $714 \mathrm{mg} / \mathrm{g}$. 当 MB 的浓度低于 250 $\mathrm{mg} / \mathrm{L}$ 时, 吸附效率高于 $99 \%$. 另外, 低温和高 $\mathrm{pH}$ 能够加 快 $\mathrm{GO}$ 对 $\mathrm{MB}$ 的吸附进程和效率. 当MB的浓度较高时, 在溶液中溶解离子和有机物也能够加快对其的吸附.

以上研究表明, GO在吸附染料时具有良好的吸 附性能. 通过对 GO进行不同程度的还原, 研究了 RGO的吸附性能, Liu等人 ${ }^{[60]}$ 用水合肼将 $\mathrm{GO}$ 还原得到 $\mathrm{RGO}$, 研究了 $\mathrm{pH}$ 、接触时间、温度和剂量对水中 $\mathrm{MB}$ 的吸附性能, 在 $293 \mathrm{~K}$ 时最大吸附量可达 $153.85 \mathrm{mg} / \mathrm{g}$. Sun等人 ${ }^{[61]}$ 用亚硫酸钠原位还原 GO得到RGO, 可以 增强对水中四呋啶橙的吸附能力. 在相同的条件下, 未还原的 $\mathrm{GO}$ 的吸附量仅为 $1.4 \mathrm{~g} / \mathrm{g}$, 还原后 RGO的吸 
附能量为 $3.3 \mathrm{~g} / \mathrm{g}$. 另有研究对比了 $\mathrm{GO}$ 和 $\mathrm{RGO}$ 对水中 $\mathrm{MB}, \mathrm{RhB}$, 甲基紫和橙黄 $\mathrm{G}$ 的吸附能力, $\mathrm{MB}$ 和甲基紫 是阳离子染料, $\mathrm{RhB}$ 和橙黄 $\mathrm{G}$ 是阴离子染料, 而 $\mathrm{GO}$ 含 有多种含氧官能团, 分散在水中带负电, 能够吸附水 中的阳离子染料, 抑制对阴离子染料的吸附. 当 $\mathrm{GO}$ 还原成RGO时, RGO的负电荷减少, 但仍具有高比表 面积, 对阴离子染料有很好的吸附性能 ${ }^{[62]}$.

综上所述, 石墨烯和 $\mathrm{GO}$ 对水中的染料具有优异 的吸附能力, 但在吸附过程中仍存在一定的局限性. 因此, 有研究尝试对石墨烯和 GO进行改性和复合来 提高其吸附能力. Sui等人 ${ }^{[63]}$ 制备了石墨烯-CNT复合 气凝胶, 这种复合材料轻便、导电率高、比表面积大 且为多孔结构, 对 $R h B$ 和 $M B$ 的吸附量分别为 145.9 和 $190.9 \mathrm{mg} / \mathrm{g}$. 复合气凝胶在水净化、金属盐、重金属 离子和有机染料的去除领域具有潜在应用. Xie等 人 $^{[64]}$ 通过一种简单有效的化学方法制备了超顺磁 $\mathrm{GO}-\mathrm{Fe}_{3} \mathrm{O}_{4}$ 复合材料, 测试对 $\mathrm{MB}$ 和中性红(NR)的吸附 能力, 结果表明, $\mathrm{GO}-\mathrm{Fe}_{3} \mathrm{O}_{4}$ 复合材料对 $\mathrm{MB}$ 和 $\mathrm{NR}$ 的吸 附量分别为 167.2 和 $171.3 \mathrm{mg} / \mathrm{g}$. 另有研究合成了石 墨烯 $-\mathrm{Fe}_{3} \mathrm{O}_{4}$ 磁性复合材料, 分别对溶液中的 $\mathrm{MB}$ 和刚 果红(CR)进行吸附, 发现对 $\mathrm{MB}$ 和 CR 的吸附量可达 45.27 和 $33.66 \mathrm{mg} / \mathrm{g}^{[65,66]}$, 吸附能力较好. 磁性复合材 料比 GO具有更高的热稳定性, 且可以在外磁场的作 用下将吸附剂分离回收利用, 是一种优良的吸附废 水中染料的吸附剂. Cheng等人 ${ }^{[67]}$ 制备了三维聚壳糖石墨烯复合材料, 具有高比表面积 $\left(603.2 \mathrm{~m}^{2} / \mathrm{g}\right)$ 和独 特的孔隙, 用于去除水溶液中的活性黑 5(RB5). 当 RB5的初始浓度为 $1.0 \mathrm{mg} / \mathrm{L}$ 时, 去除率高达 $97.5 \%$.

\section{3 对有机污染物的吸附}

有机污染物广泛存在于各种废水中, 严重影响 人类的健康. 目前, 利用石墨烯吸附废水中的酚 类 ${ }^{[68 \sim 72]}$ 、油污 ${ }^{[73 ~ 76]}$ 、抗生素 ${ }^{[77,78]}$ 、农药 ${ }^{[79]}$ 和腐植酸 ${ }^{[80]}$ 等有机物逐渐引起人们的关注.

对石墨烯和GO对酚类有机物的吸附机理的研究 表明, 疏水作用、 $\pi-\pi$ 键交互作用、氢键、共价键和 静电相互作用会影响石墨烯和GO对有机物的吸附能 力. Pei等人 ${ }^{[68]}$ 分析了石墨烯和氧化石墨烯吸附 $1,2,4-$ 三氯苯 $(\mathrm{TCB}) 、 2,4,6$-三氯苯酚 $(\mathrm{TCP}) 、 2$ - 菜酚和萗 (NAPH)的吸附机理. 结果表明, 在吸附过程中除了 疏水相互作用, 还存在某些特定的相互作用. 例如, 石墨烯吸附TCP、TCB和 2 - 荎酚时主要通过 $\pi-\pi$ 键的相
互作用, 而 GO对 TCP和2-菜酚的较高吸附性能, 主 要由于 TCP的差基和 $\mathrm{GO}$ 上的含氧官能团形成氢键的 相互作用. $\mathrm{Xu}$ 等人 ${ }^{[69]}$ 研究了不同条件下(如温度、 $\mathrm{pH}$ 等)石墨烯对水溶液中双酚 $\mathrm{A}(\mathrm{BPA})$ 的吸附能力. 由 Langmuir等温曲线可知最大吸附量在 $302.15 \mathrm{~K}$ 时为 $182 \mathrm{mg} / \mathrm{g}$, 是目前碳吸附材料中最高的. 石墨烯良好 的吸附性能主要源于石墨烯单层原子的 $\mathrm{sp}^{2}$ 杂化, 在 吸附过程中主要通过 $\pi-\pi$ 键和氢键的作用.

另有研究对石墨烯吸附苯酚的热力学和动力学 平衡进行了测试. $\mathrm{Li}$ 等人 ${ }^{[70]}$ 测试了不同 $\mathrm{pH}$ 、温度、剂 量、接触时间对吸附性能的影响. 当苯酚初始浓度为 $50 \mathrm{mg} / \mathrm{g} 、 \mathrm{pH}$ 为 6.3 、温度为 $285 \mathrm{~K}$ 时, 最大吸附量为 $28.26 \mathrm{mg} / \mathrm{g}$. 热力学吸附研究表明, 石墨烯吸附苯酚 是一种自发的吸热过程. 动力学吸附研究表明, 石墨 烯吸附苯酚符合准二级动力学模型. Zhao等人 ${ }^{[71,72]}$ 制 备了高度分散的磺化石墨烯纳米片来吸附水中的芳 香污染物, 磺化石墨烯对水中的荎和1-荎酚的吸附 量约为 $2.3 \sim 2.4 \mathrm{mmol} / \mathrm{g}$. 对吸附 1 -萘酚的动力学和热 力学吸附的研究结果表明, 1-荟酚更倾向于以低活化 能堆叠在石墨烯纳米片表面, 吸附过程是一个自发 的吸热过程, 且和非键相互作用理论吻合得很好. 在 $293.15 \mathrm{~K}$ 时吸附量高达 $2.3 \mathrm{mmol} / \mathrm{g}$, 在 313.15 和 $333.15 \mathrm{~K}$ 时, 吸附量约为 $6.4 \mathrm{mmol} / \mathrm{g}$, 是目前纳米材 料中吸附能力最高的. 磺化石墨烯良好的吸附性能 在处理水中芳香化合物方面具有突出的优势.

石墨烯除了吸附酚类有机物外, 对油污也有良 好的吸附性能. Bi等人 ${ }^{[73]}$ 制备了一种形状可塑多孔且 可循环利用的石墨烯海绵, 不仅对石油和脂肪有着 高效的吸附能力, 而且对有毒溶剂甲苯和氯仿的吸 附量可达自身重量的 86 倍, 高于传统吸附材料的 10 倍. Zhao等人 ${ }^{[74]}$ 自组装了一种新型的孔径可调、力学 性能好、可重复利用的石墨烯海绵, 可以有效地吸附 油污、染料和有机溶剂, 对柴油的吸附量为 $129 \mathrm{~g} / \mathrm{g}$. 通过研究石墨烯海绵结构和吸附性能之间的关系, 发现油污的吸附主要与比表面积有关. Wang 等人 ${ }^{[75]}$ 自组装了三维石墨烯, 对洜油、煤油和石蜡油等具有 良好的吸附性能, 如图8(a) (c)所示. 超轻N掺杂的三 维石墨烯框架对油污和其它有机污染物也有良好的 吸附性能, 对汽油的的吸附量高达 $277 \mathrm{~g} / \mathrm{g}^{[76]}$, 如图 $8(\mathrm{~d})$ 所示. 由上可以看出, 在处理油污污染物时多为 三维石墨烯, 这是由于三维石墨烯不仅有高效的吸 附能力而且力学性能好, 大多可循环利用. 三维石墨 
(a)

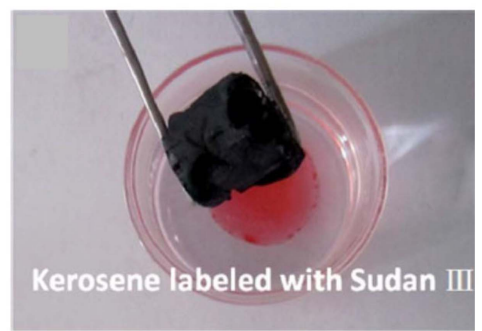

(c)

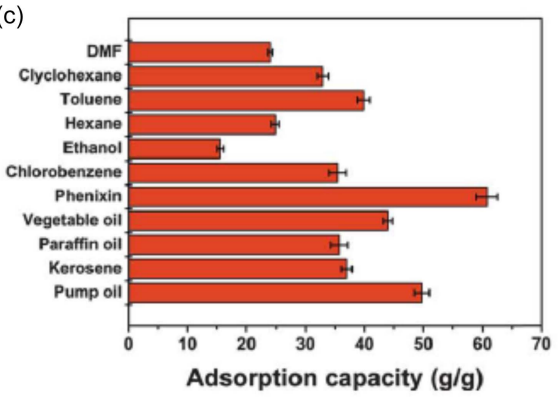

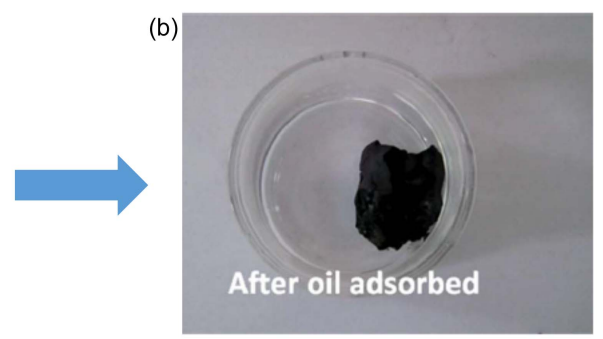

(d)

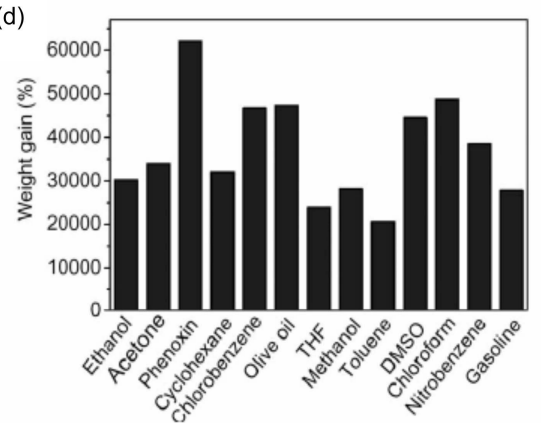

图 8 (网络版彩色)(a)和(b)石墨烯海绵吸附被苏丹 3 标记的煤油; (c) 石墨烯海绵对不同种类油污的吸附量 ${ }^{[75]}$; (d) 对不同有机污染物的吸附 能力 ${ }^{[76]}$

Figure 8 (Color online) (a), (b) Photograph of the kerosene adsorption process of graphene sponge. The kerosene was labeled with Sudan III for clear observation; (c) adsorption capacities for different oils and organic solvents at saturated state ${ }^{[75]}$; (d) adsorption efficiency in terms of weight gain ${ }^{[76]}$

烯多孔、密度小、疏水等优点使得其在处理工业油污 时具有很好的应用潜力.

此外, 另有研究测试了石墨烯纳米材料对抗生 素、农药和腐植酸等的吸附. 例如, Gao等人 ${ }^{[77]}$ 采用 GO吸附水中的四环素、土霉素和强力霉素, 最高吸 附量分别为 313,212 和 $398 \mathrm{mg} / \mathrm{g}$. 四环素吸附在GO表 面上是通过 $\pi-\pi$ 键的作用和阳离子 $-\pi$ 的结合. Zhao等 人 $^{[78]}$ 制备了多孔 GO-壳聚糖气凝胶来吸附四环素, 吸附量高达 $1470 \mathrm{mg} / \mathrm{g}$, 是吸附四环素最有效的吸附 剂之一. Maliyekkal等人 ${ }^{[79]}$ 研究了 GO 和RGO对农药 如毒死蜱、硫单和马拉硫磷的吸附, 发现RGO的吸附 性能高于 GO, 吸附量分别高达 1200,1100 和 800 $\mathrm{mg} / \mathrm{g}$. 研究还发现, 吸附过程对 $\mathrm{pH}$ 和溶液中的离子 并不敏感. 根据第一性原理密度泛函理论计算发现, 石墨烯-水-农药在相互作用的过程中, 水起到媒介的 作用, 石墨烯和农药的接触是很微弱的. Hartono等 人 $^{[80]}$ 制备了层状 GO来吸附水中的腐植酸, 发现 $\mathrm{GO}$ 石墨烯的吸附性能远高于石墨和活性炭, 最大吸附 量达 $190 \mathrm{mg} / \mathrm{g}$, 但随着pH增大吸附量将减少.

\section{3 结论与展望}

综上所述, 石墨烯不仅对气体、水合溶液中的离
子具有独特的传质行为，而且对污染有着良好的吸 附性能. 石墨烯在海水淡化领域相比于其它膜有许 多优点, $\mathrm{NG}$ 薄膜其超薄的厚度理论上对气体分离、海 水淡化处理领域有着非常诱人的应用前景, 但是, 在 实际应用中, $\mathrm{NG}$ 不仅需要较高的强度, 还需要精确 的打孔技术. 目前合成的石墨烯大多为多晶结构, 强 度较差, 不适合应用于高压状态. 另外, 传统的打孔 技术仍存在诸多不足，不仅打出的孔径大小不均匀, 且孔径过于集中某一部位, 进一步削弱 NG的强度. 因此, 目前的 $\mathrm{NG}$ 薄膜应用到实际还存着一定的困难.

而对于层状的 GO薄膜, Joshi等人 ${ }^{[34]}$ 报道层状 $\mathrm{GO}$ 浸人水中可以充当分子篮, $\mathrm{GO}$ 片之间会形成 $0.9 \mathrm{~nm}$ 的毛细通道, 这种通道能够阻挡所有水合半径大于 $0.45 \mathrm{~nm}$ 的离子, 而半径小于 $0.45 \mathrm{~nm}$ 的离子渗透速率 比自由扩散的速率高出数千倍, 因此层状 $\mathrm{GO}$ 薄膜在 海水淡化等领域有较好的潜在应用. 基于当前 $\mathrm{GO}$ 薄 膜较低的脱盐率, $\mathrm{Mi}$ 等人 ${ }^{[35]}$ 报道, 在海水淡化领域, 需要将 $\mathrm{GO}$ 的层间距减小至 $0.7 \mathrm{~nm}$ 以下 $\left(\mathrm{Na}^{+}\right.$水合直径 为 $0.72 \mathrm{~nm}$ ), 可以通过对 $\mathrm{GO}$ 进行不同程度的还原或 插人二维纳米薄片来控制GO层间距的大小从而达到 脱盐. 如何控制 GO薄膜的层间距使水分子畅通无阻 的通过而盐离子被截留是 $\mathrm{GO}$ 薄膜应用到实际领域的 
关键和今后研究目标所在.

石墨烯材料对重金属离子、染料和有机物污染物 都具有很好的吸附能力. 但石墨烯的疏水性使其在 水溶液中的吸附受到一定的限制. GO作为石墨烯的 一种衍生物, 表面含有大量的羟基、羧基、环氧基等 含氧基团, 亲水性较好, 表面的负电荷较高, 所以常 用于处理带正电的的污染物. 另外, 易于分离、环境 友好的磁性石墨烯复合材料和不易团聚、易于分离的
三维的石墨烯复合材料在水处理中的应用具有更广 阔的发展前景. 尽管如此, 目前, 石墨烯复合材料广 泛应用于水处理还面临着诸多问题. 首先, 现有研究 主要基于模型污染物的基础工作，而面向实际应用 的研究却很鲜见. 其次, 石墨烯对污染物吸附的选择 性较差. 另外, 如何大批量、低成本制备石墨烯吸附 材料并实现高效回收仍是石墨烯吸附材料实际应用 的关键瓶颈.

\section{参考文献}

1 Baughman R H, Zakhidov A A, de Heer W A. Carbon nanotubes-the route toward applications. Science, 2002, 297: 787-792

2 Lee C, Wei X, Kysar J W, et al. Measurement of the elastic properties and intrinsic strength of monolayer graphene. Science, 2008, 321: 385-388

3 Balandin A A, Ghosh S, Bao W, et al. Superior thermal conductivity of single-layer graphene. Nano Lett, 2008, 8: 902-907

4 Hou X, Guo W, Jiang L. Biomimetic smart nanopores and nanochannels. Chem Soc Rev, 2011, 40: 2385-2401

5 Bocquet L, Charlaix E. Nanofluidics, from bulk to interfaces. Chem Soc Rev, 2010, 39: 1073-1095

6 Zhao G, Wen T, Chen C, et al. Synthesis of graphene-based nanomaterials and their application in energy-related and environmental-related areas. RSC Adv, 2012, 2: 9286-9303

7 Bunch J S, Verbridge S S, Alden J S, et al. Impermeable atomic membranes from graphene sheets. Nano Lett, 2008, 8: 2458-2462

$8 \mathrm{Du} \mathrm{H}$, Li J, Zhang J, et al. Separation of hydrogen and nitrogen gases with porous graphene membrane. J Phys Chem C, 2011, 115: 23261-23266

9 Sint K, Wang B, Král P. Selective ion passage through functionalized graphene nanopores. J Am Chem Soc, 2008, 130: 16448-16449

10 Cohen-Tanugi D, Grossman J C. Water desalination across nanoporous graphene. Nano Lett, 2012, 12: 3602-3608

$11 \mathrm{He}$ Z, Zhou J, Lu X, et al. Bioinspired graphene nanopores with voltage-tunable ion selectivity for $\mathrm{Na}^{+}$and $\mathrm{K}^{+}$. ACS Nano, 2013, 7: 10148-10157

12 Koenig S P, Wang L, Pellegrino J, et al. Selective molecular sieving through porous graphene. Nat Nanotechnol, 2012, 7: 728-732

13 Celebi K, Buchheim J, Wyss R M, et al. Ultimate permeation across atomically thin porous graphene. Science, 2014, 344: 289-292

14 O'Hern S C, Stewart C A, Boutilier M S H, et al. Selective molecular transport through intrinsic defects in a single layer of CVD graphene. ACS Nano, 2012, 6: 10130-10138

15 O Hern S C, Boutilier M S H, Idrobo J, et al. Selective ionic transport through tunable subnanometer pores in single-layer graphene membranes. Nano Lett, 2014, 14: 1234-1241

16 Surwade S P, Smirnov S N, Vlassiouk I V, et al. Water desalination using nanoporous single-layer graphene. Nat Nanotechnol, 2015, 10: 459-464

17 Li X, Cai W, An J, et al. Large-Area synthesis of high-quality and uniform graphene films on copper foils. Science, 2009, 324: 1312-1314

18 Hummers W S, Offeman R E. Preparation of graphite oixde. J Am Chem Soc, 1958, 80: 1339

19 Loh K P, Bao Q, Eda G, et al. Graphene oxide as a chemically tunable platform for optical applications. Nat Chem, 2010, 2: 1015-1024

20 Schniepp H C, Li J L, Mcallister M J, et al. Functionalized single graphene sheets derived from splitting graphite oxide. J Phys Chem B, 2006, 110: 8535-8539

21 Gomez-Navarro C, Weitz R T, Bittner A M, et al. Electronic transport properties of individual chemically reduced graphene oxide sheets. Nano Lett, 2007, 7: 3499-3503

22 Mattevi C, Eda G, Agnoli S, et al. Evolution of electrical, chemical, and structural properties of transparent and conducting chemically derived graphene thin films. Adv Funct Mater, 2009, 19: 2577-2583

23 Eda G, Fanchini G, Chhowalla M. Large-area ultrathin films of reduced graphene oxide as a transparent and flexible electronic material. Nat Nanotechnol, 2008, 3: 270-274

24 Robinson J T, Zalalutdinov M, Baldwin J W, et al. Wafer-scale reduced graphene oxide films for nanomechanical devices. Nano Lett, 2008, 8: 3441-3445 
25 Robinson J T, Perkins F K, Snow E S, et al. Reduced graphene oxide molecular sensors. Nano Lett, 2008, 8: 3137-3140

26 Nair R R, Wu H A, Jayaram P N, et al. Unimpeded permeation of water through helium-leak-tight graphene-based membranes. Science, 2012, 335: 442-444

27 Boukhvalov D W, Katsnelson M I, Son Y. Origin of anomalous water permeation through graphene oxide membrane. Nano Lett, 2013, 13: 3930-3935

28 Kim H W, Yoon H W, Yoon S, et al. Selective gas transport through few-layered graphene and graphene oxide membranes. Science, 2013, 342: 91-95

29 Li H, Song Z, Zhang X, et al. Ultrathin, molecular-sieving graphene oxide membranes for selective hydrogen separation. Science, 2013, 342: 95-98

30 Sun P, Liu H, Wang K, et al. Ultrafast liquid water transport through graphene-based nanochannels measured by isotope labelling. Chem Commun, 2015, 51: 3251-3254

31 Sun P, Zhu M, Wang K, et al. Selective ion penetration of graphene oxide membranes. ACS Nano, 2013, 7: 428-437

32 Sun P, Liu H, Wang K, et al. Selective ion transport through functionalized graphene membranes based on delicate ion-graphene interactions. J Phys Chem C, 2014, 118: 19396-19401

33 Sun P, Zheng F, Zhu M, et al. Selective trans-membrane transport of alkali and alkaline earth cations through graphene oxide membranes based on cation- $\pi$ interactions. ACS Nano, 2014, 8: 850-859

34 Joshi R K, Carbone P, Wang F C, et al. Precise and ultrafast molecular sieving through graphene oxide membranes. Science, 2014, 343: $752-754$

35 Mi B. Graphene oxide membranes for ionic and molecular sieving. Science, 2014, 343: 740-742

$36 \mathrm{Hu}$ M, Mi B. Enabling graphene oxide nanosheets as water separation membranes. Environ Sci Technol, 2013, 47: 3715-3723

37 Han Y, Xu Z, Gao C. Ultrathin graphene nanofiltration membrane for water purification. Adv Funct Mater, 2013, 23: 3693-3700

38 Sun P, Chen Q, Li X, et al. Highly efficient quasi-static water desalination using monolayer graphene oxide/titania hybrid laminates. NPG Asia Mater, 2015, 7: e162

39 Su Y, Kravets V G, Wong S L, et al. Impermeable barrier films and protective coatings based on reduced graphene oxide. Nat Commun, 2014, 5: 4843

40 Liu H, Wang H, Zhang X. Facile fabrication of freestanding ultrathin reduced graphene oxide membranes for water purification. Adv Mater, 2015, 27: 249-254

41 Huang Z, Zheng X, Lv W, et al. Adsorption of lead (II) Ions from aqueous solution on low-temperature exfoliated graphene nanosheets. Langmuir, 2011, 27: 7558-7562

42 Leng Y, Guo W, Su S, et al. Removal of antimony (III) from aqueous solution by graphene as an adsorbent. Chem Eng J, 2012, 211-212: 406-411

43 Yang S, Chang Y, Wang $\mathrm{H}$, et al. Folding/aggregation of graphene oxide and its application in $\mathrm{Cu}^{2+}$ removal. $\mathrm{J} \mathrm{Colloid}$ Interface Sci, 2010, 351: 122-127

44 Wang H, Yuan X, Wu Y, et al. Adsorption characteristics and behaviors of graphene oxide for Zn(II) removal from aqueous solution. Appl Surf Sci, 2013, 279: 432-440

45 Li Z, Chen F, Yuan L, et al. Uranium(VI) adsorption on graphene oxide nanosheets from aqueous solutions. Chem Eng J, 2012, 210: $539-546$

46 Zhao G, Wen T, Yang X, et al. Preconcentration of U(VI) ions on few-layered graphene oxide nanosheets from aqueous solutions. Dalton Trans, 2012, 41: 6182-6188

47 Zhao G, Li J, Ren X, et al. Few-layered graphene oxide nanosheets as superior sorbents for heavy metal ion pollution management. Environ Sci Technol, 2011, 45: 10454-10462

48 Liu L, Liu S, Zhang Q, et al. Adsorption of Au(III), Pd(II), and Pt(IV) from aqueous solution onto graphene oxide. J Chem Eng Data, 2013, 58: 209-216

49 Sitko R, Turek E, Zawisza B, et al. Adsorption of divalent metal ions from aqueous solutions using graphene oxide. Dalton Trans, 2013, 42: 5682-5689

50 Zhang Y, Yan $\mathrm{L}, \mathrm{Xu} \mathrm{W}$, et al. Adsorption of $\mathrm{Pb}(\mathrm{II})$ and $\mathrm{Hg}(\mathrm{II})$ from aqueous solution using magnetic $\mathrm{CoFe}_{2} \mathrm{O}_{4}{ }^{-}$reduced graphene oxide. $\mathrm{J}$ Mol Liq, 2014, 191: 177-182

51 Liu M, Chen C, Hu J, et al. Synthesis of magnetite/graphene oxide composite and application for Cobalt(II) removal. J Phys Chem C, 2011, 115: 25234-25240

52 Chen M, Sun Y, Huo C, et al. Akaganeite decorated graphene oxide composite for arsenic adsorption/removal and its proconcentration at ultra-trace level. Chemosphere, 2015, 130: 52-58 
53 Madadrang C J, Kim H Y, Gao G, et al. Adsorption behavior of EDTA-graphene oxide for Pb (II) removal. ACS App Mater Inter, 2012, 4: $1186-1193$

54 Dong S, Dou X, Mohan D, et al. Synthesis of graphene oxide/schwertmannite nanocomposites and their application in Sb(V) adsorption from water. Chem Eng J, 2015, 270: 205-214

55 Mi X, Huang G, Xie W, et al. Preparation of graphene oxide aerogel and its adsorption for $\mathrm{Cu}^{2+}$ ions. Carbon, 2012, 50: 4856-4864

$56 \mathrm{Li} \mathrm{W}, \mathrm{Gao} \mathrm{S}, \mathrm{Wu}$ L, et al. High-density three-dimension graphene macroscopic objects for high-capacity removal of heavy metal ions. Sci Rep, 2013, 3: 2125

57 Lei Y, Chen F, Luo Y, et al. Synthesis of three-dimensional graphene oxide foam for the removal of heavy metal ions. Chem Phys Lett, 2014, 593: 122-127

58 Bradder P, Ling S K, Wang S, et al. Dye adsorption on layered graphite oxide. J Chem Eng Data, 2011, 56: 138-141

59 Yang S, Chen S, Chang Y, et al. Removal of methylene blue from aqueous solution by graphene oxide. J Colloid Interface Sci, 2011, 359: 24-29

60 Liu T, Li Y, Du Q, et al. Adsorption of methylene blue from aqueous solution by graphene. Colloid Surface B, 2012, 90: 197-203

61 Sun L, Yu H, Fugetsu B. Graphene oxide adsorption enhanced by in situ reduction with sodium hydrosulfite to remove acridine orange from aqueous solution. J Hazard Mater, 2012, 203-204: 101-110

62 Ramesha G K, Vijaya Kumara A, Muralidhara H B, et al. Graphene and graphene oxide as effective adsorbents toward anionic and cationic dyes. J Colloid Interface Sci, 2011, 361: 270-277

63 Sui Z Y, Meng Q, Zhang X T, et al. Green synthesis of carbon nanotube-graphene hybrid aerogels and their use as versatile agents for water purification. J Mater Chem, 2012, 22: 8767-8771

64 Xie G, Xi P, Liu H, et al. A facile chemical method to produce superparamagnetic graphene oxide- $\mathrm{Fe}_{3} \mathrm{O}_{4}$ hybrid composite and its application in the removal of dyes from aqueous solution. J Mater Chem, 2012, 22: 1033-1039

65 Wang C, Feng C, Gao Y, et al. Preparation of a graphene-based magnetic nanocomposite for the removal of an organic dye from aqueous solution. Chem Eng J, 2011, 173: 92-97

66 Yao Y, Miao S, Liu S, et al. Synthesis, characterization, and adsorption properties of magnetic $\mathrm{Fe}_{3} \mathrm{O}_{4} @$ graphene nanocomposite. Chem Eng J, 2012, 184: 326-332

67 Cheng J, Du J, Zhu W. Facile synthesis of three-dimensional chitosan-graphene mesostructures for reactive black 5 removal. Carbohyd Polym, 2012, 88: 61-67

68 Pei Z, Li L, Sun L, et al. Adsorption characteristics of 1,2,4-trichlorobenzene, 2,4,6-trichlorophenol, 2-naphthol and naphthalene on graphene and graphene oxide. Carbon, 2013, 51: 156-163

69 Xu J, Wang L, Zhu Y. Decontamination of bisphenol a from aqueous solution by graphene adsorption. Langmuir, 2012, 28: 8418-8425

$70 \mathrm{Li} \mathrm{Y,} \mathrm{Du} \mathrm{Q,} \mathrm{Liu} \mathrm{T,} \mathrm{et} \mathrm{al.} \mathrm{Equilibrium,} \mathrm{kinetic} \mathrm{and} \mathrm{thermodynamic} \mathrm{studies} \mathrm{on} \mathrm{the} \mathrm{adsorption} \mathrm{of} \mathrm{phenol} \mathrm{onto} \mathrm{graphene.} \mathrm{Mater} \mathrm{Res} \mathrm{Bull,}$ 2012, 47: 1898-1904

71 Zhao G, Jiang L, He Y, et al. Sulfonated graphene for persistent aromatic pollutant management. Adv Mater, 2011, 23: 3959-3963

72 Zhao G, Li J, Wang X. Kinetic and thermodynamic study of 1-naphthol adsorption from aqueous solution to sulfonated graphene nanosheets. Chem Eng J, 2011, 173: 185-190

73 Bi H, Xie X, Yin K, et al. Spongy graphene as a highly efficient and recyclable sorbent for oils and organic solvents. Adv Funct Mater, 2012, 22: 4421-4425

74 Zhao J, Ren W, Cheng H. Graphene sponge for efficient and repeatable adsorption and desorption of water contaminations. J Mater Chem, 2012, 22: 20197

75 Wang J, Shi Z, Fan J, et al. Self-assembly of graphene into three-dimensional structures promoted by natural phenolic acids. J Mater Chem, 2012, 22: 22459

76 Zhao Y, Hu C, Hu Y, et al. A versatile, ultralight, nitrogen-doped graphene framework. Angew Chem, 2012, 124: 11533-11537

77 Gao Y, Li Y, Zhang L, et al. Adsorption and removal of tetracycline antibiotics from aqueous solution by graphene oxide. J Colloid Interface Sci, 2012, 368: 540-546

78 Zhao L, Dong P, Xie J, et al. Porous graphene oxide-chitosan aerogel for tetracycline removal. Mater Res Express, 2014, 1: 015601-0156012

79 Maliyekkal S M, Sreeprasad T S, Krishnan D, et al. Graphene: A reusable substrate for unprecedented adsorption of pesticides. Small, 2013, 9: 273-283

80 Hartono T, Wang S, Ma Q, et al. Layer structured graphite oxide as a novel adsorbent for humic acid removal from aqueous solution. J Colloid Interface Sci, 2009, 333: 114-119 


\title{
Applications of graphene-based materials in water treatment: mass transport and pollutants adsorption properties
}

\author{
DENG Hui ${ }^{1,2}$, SUN PengZhan ${ }^{2}$, ZHANG YingJiu ${ }^{1} \&$ ZHU HongWei ${ }^{2}$ \\ ${ }^{1}$ Department of Physics, Zhengzhou University, Zhengzhou 450052, China; \\ ${ }^{2}$ School of Materials Science and Engineering, State Key Laboratory of New Ceramics and Fine Processing, Tsinghua University, Beijing 100084, \\ China
}

Graphene-based nanomaterials have been used as separation membranes and adsorbents due to its unique two-dimensional single atomic layer structure, large specific surface area and excellent properties. In this paper, we first review gas, water and ions mass transport phenomenon through nanoporous graphene membranes and layered structures of graphene oxides. The development of nanoporous graphene has been limited by the current preparation technique and immature hole-drilling technology; while the laminated graphene oxide membranes have a great application potential in the field of water purification due to its simple, low-cost preparation methods, high permeability and selectivity. Then, we review the adsorption behaviors of heavy metal ions, dyes and organic pollutants in aqueous solution with graphene-based nanomaterials. The interaction mechanism between functional groups on graphene-based materials and pollutants has been analyzed. Finally, we prospect the opportunities and challenges of graphene-based nanomaterials in environmental applications, such as membrane separation, water desalination and pollutants removal.

grapheme, graphene oxide, mass transport, adsorption, water treatment

doi: 10.1360/N972015-00709 